\title{
The relationship of syphilis to small vessel stroke in the absence of neurosyphilis: real or imaginary?
}

A relação da sífilis com o acidente vascular cerebral na ausência de neurossífilis: real ou imaginário? Cody L. NATHAN ${ }^{1}$, Joseph R. BERGER ${ }^{1}$

'University of Pennsylvania, Perelman School of Medicine, Department of Neurology, Philadelphia PA, USA.

Cody L. NATHAN (D https://orcid. org/0000-0001-9407-3427; Joseph R. BERGER (ID https://orcid. org/0000-0002-3821-1749 Correspondence: Joseph R. Berger;E-mail:joseph.berger@ pennmedicine.upenn.edu

Conflict of Interest: Cody L. Nathan: None to disclose. In the last 3 years, Dr. Berger reports grants to the institution on his behalf from Biogen and Genentech/Roche and personal fees from Amgen, Alkermes, AstraZeneca, Bayer, Biogen, Dr. Reddy, Encycle, Excision-Bio, Genentech/ Roche, Genzyme, Inhibikase, Merck, Millennium/Takeda, Novartis, Serono, and Shire.

Authors' contributions: CLN: drafting the article. JRB: critical revision of the article.

Received on January 17, 2021; Accepted on February 4, 2021.

\section{(cc) BY}

I n a prospective study from Universidade Federal do Paraná published in this issue of Revista Arquivos de Neuro-Psiquiatria, Ferreira and colleagues ${ }^{1}$ demonstrated an association between small vessel stroke and positive syphilis serology after excluding patients with cerebrospinal fluid (CSF) findings consistent with neurosyphilis. The authors argue that this association is likely a consequence of endothelial dysfunction resulting from Treponema pallidum infection.

Abnormalities of the CSF in early syphilis had been commonly recognized in multiple studies dating from the early $20^{\text {th }}$ century and indicated that T. pallidum frequently invaded the central nervous system during the hematogenous dissemination of early stages of infection. The presence of these CSF abnormalities was recognized to increase the risk of the subsequent development of symptomatic neurosyphilis². Modern technology using polymerase chain reaction has confirmed that the organism is present in the CSF during primary and secondary syphilis in more than $25 \%$ of infected individuals ${ }^{3}$, though the rates of clinically recognized neurosyphilis in untreated syphilis, as revealed by the Oslo and Tuskegee studies, are substantially lower ${ }^{4}$. One form of neurosyphilis, referred to as meningovascular syphilis, usually occurs 6 to 7 years after initial infection, but may manifest in up to 6 months ${ }^{5}$. Typically, in meningovascular syphilis, large and medium sized intracranial arteries are affected; a condition referred to as Huebner's endarteritis. Histopathological examination reveals thickening of the arterial walls with separation of the intima concentrically or eccentrically from the internal elastic lamina by an inflammatory infiltrate comprised of monocytes and lymphocytes and often plasma cells and eosinophils. Inflammation of small vessels with intimal fibrosis leading to stroke is also a recognized form of meningovascular syphilis, although less common. Small vessel involvement is referred to as Nissl-Alzheimer endarteritis. There is evidence to suggest that, even in the absence of clinical meningovascular syphilis, cerebral blood flow abnormalities exist potentially due to a subclinical inflammatory state ${ }^{6,7}$.

In this study, the authors propose that individuals with first-ever ischemic stroke (FEIS) secondary to small vessel disease, statistically, are more often of positive syphilis serology in the absence of neurosyphilis than individuals with other forms of ischemic stroke. The authors performed a retrospective analysis of patients with FIES and separated them into those with positive and negative syphilis serologic testing. The mechanism of stroke (according to the TOAST and ASCOD phenotype) in each cohort was determined by the combination of vessel imaging, brain imaging, and cardiac evaluation. Based on the TOAST criteria, there was a higher percentage of patients (31 versus 14\%) with positive syphilis serology testing who had a stroke secondary to small vessel disease. Similarly, based on the ASCOD phenotyping, there was a higher percentage (28 versus $9 \%$ ) of small vessel disease strokes in patients who tested positive for syphilis.

The increased incidence of stroke secondary to small vessel disease in patients with syphilis was ascribed to an increased inflammation of endothelial cells within small vessels. More specifically, the authors propose that proteins expressed by T. pallidum led to an upregulation of the expression of intercellular adhesion molecule-1 (ICAM-1), E-selectin, and monocyte chemoattractant protein-1 (MCP-1). The upregulation and increased gene transcription of these molecules resulted in an increased migration of monocytes to endothelial cells 
and increased membrane permeability, ultimately increasing the likelihood of small vessel occlusion.

One shortcoming of this study is the small sample size of 269 patients who met the inclusion criteria. A retrospective study by Xiang et al. ${ }^{7}$ in 2020 comparing stroke patients with positive $(n=668)$ and negative syphilis serology $(n=785)$ found no significant difference in stroke secondary to smallvessel occlusions $(\mathrm{p}=0.637)$. Thus, repeating this study with a larger patient population may yield different results. Another limitation to the study is that the patients' HIV status is not documented. There is a higher incidence of neurosyphilis, including meningovascular syphilis, in patients with HIV compared to the seronegative population ${ }^{5,8}$. HIV infection, even in the absence of neurosyphilis, is a risk factor for arterial ischemic strokes and, therefore, is a potential confounding variable contributing to small vessel disease ${ }^{9}$.

It is possible that infection with T. pallidum in the absence of neurosyphilis leads to increased inflammation within small vessels, causing stroke. Larger patient population and study cohort would be needed to substantiate this theory.

\section{References}

1. Ferreira MG, Scavasine VC, Breda GL, Almeida BMM, Zérola VHF, Lange MC. Small vessel occlusion and syphilis in patients with first ever ischemic stroke. Arq Neuro-Psiquiatr. 2020. In Press.

2. Moore JE, Hopkins HH. Asymptomatic neurosyphilis. IV. The prognosis of early and late asymptomatic neurosyphilis. JAMA. 1930 Nov;95(22):1637-41. https://doi.org/10.1001/ jama.1930.02720220007003

3. Hagihara M, Yamagishi Y, Kato H, Shibata Y, Shiota A, Sakanashi D, et al. Frequency of Treponema pallidum invasion into cerebrospinal fluid in primary or secondary early-stage syphilis. J Infect Chemother. 2018 May;24(5):404-6. https://doi.org/10.1016/j.jiac.2017.11.007

4. Clark EG, Danbolt N. The Oslo study of the natural history of untreated syphilis; an epidemiologic investigation based on a restudy of the Boeck-Bruusgaard material; a review and appraisal. J Chronic Dis. 1955 Sep;2(3):311-44. https://doi.org/10.1016/00219681(55)90139-9
5. Berger JR, Dean D. Neurosyphilis. Handb Clin Neurol. 2014;121:146172. https://doi.org/10.1016/B978-0-7020-4088-7.00098-5

6. Shi X, Wu J, Liu Z, Tang J, Su Y. Single photon emission CT perfusion imaging of cerebral blood flow of early syphilis patients. Chin Med J (Engl). 2003 Jul;116(7):1051-4.

7. Xiang L, Zhang T, Zhang B, Zhang C, Cui W, Yue W. Positive syphilis serology contributes to intracranial stenosis in ischemic stroke patients. Brain Behav. 2021 Jan;11(1):e01906. https://doi. org/10.1002/brb3.1906

8. Brightbill TC, Ihmeidan IH, Post MJ, Berger JR, Katz DA. Neurosyphilis in HIV-positive and HIV-negative patients: neuroimaging findings. AJNR Am J Neuroradiol. 1995 Apr;16(4):703-11.

9. Benjamin LA, Bryer A, Lucas S, Stanley A, Allain TJ, Joekes E, et al. Arterial ischemic stroke in HIV: Defining and classifying etiology for research studies. Neurol Neuroimmunol Neuroinflamm. 2016 Jun 30;3(4):e254. https://doi.org/10.1212/NXI.0000000000000254 